\title{
Can dietary supplements reduce cell-surface expression of molecules involved in organ transplantation?
}

\author{
K. Varley, B. Clark and C. Carter \\ Transplant Immunology Department, St James Hospital, Leeds LS9 7TF, UK
}

Preformed donor-relevant antibodies are a contraindication to successful renal transplantation, causing irreversible damage to the endothelial cells on the donor organ that leads to vessel obstruction and swift rejection. Such sensitised patients represent a challenge to successful renal transplantation and individual treatment plans have become necessary in order to obtain a negative cross-match. Current methods used to treat the recipient before transplant are based on the removal of donor-relevant antibodies and include the use of high-dose intravenous Ig, immunoadsorption and plasmapheresis. All methods of reducing antibody levels require immunosuppression therapies in order to halt antibody re-synthesis. Whilst significant efforts are made to control the recipient's immune system, scant regard is given to direct modulation of the donor antigens present on the transplanted organ.

In the present study a strategy was explored in which pre-conditioning of the donor, in a live donor situation, could lead to a transient reduction of human leucocyte antigen (HLA) expression at the time of transplant, thus reducing organ antigenicity. Several studies have shown that specific compounds can act as immunomodulatory agents. Whilst the use of prescription drugs in a healthy donor would be obviously inappropriate, $n-3$ fish oil (a widely-used food supplement) has been shown to have a direct effect on peripheral monocytes by inhibiting the expression of surface molecules involved in antigen presentation ${ }^{(1)}$. Although controversial, the relatively innocuous nature of fish oils indicates their potential as an acceptable immune-response modifier for use in the diet of the organ donor before transplant.

In order to quickly screen a number of potential compounds, work was undertaken to establish an in vitro screen for HLA class II expression and other relevant cell-surface molecules on human monocytic cell lines. This procedure will be used to test potential compounds (health food or dietary supplements) to assess their ability to down regulate molecules involved in antigen presentation. The pre-monocytic cell line THP-1 was analysed by two-colour flow cytometry to measure baseline expression of CD14/HLA-DR. Cells were subsequently stimulated with lipopolysaccharide (LPS) for $24 \mathrm{~h}$ or $48 \mathrm{~h}$ and analysed for CD14/HLA-DR up-regulation. A representative example showing CD14 (FL-1)/HLA-DR(FL-2) expression from unstimulated and LPS-stimulated THP-1 cells is shown in the Figure.
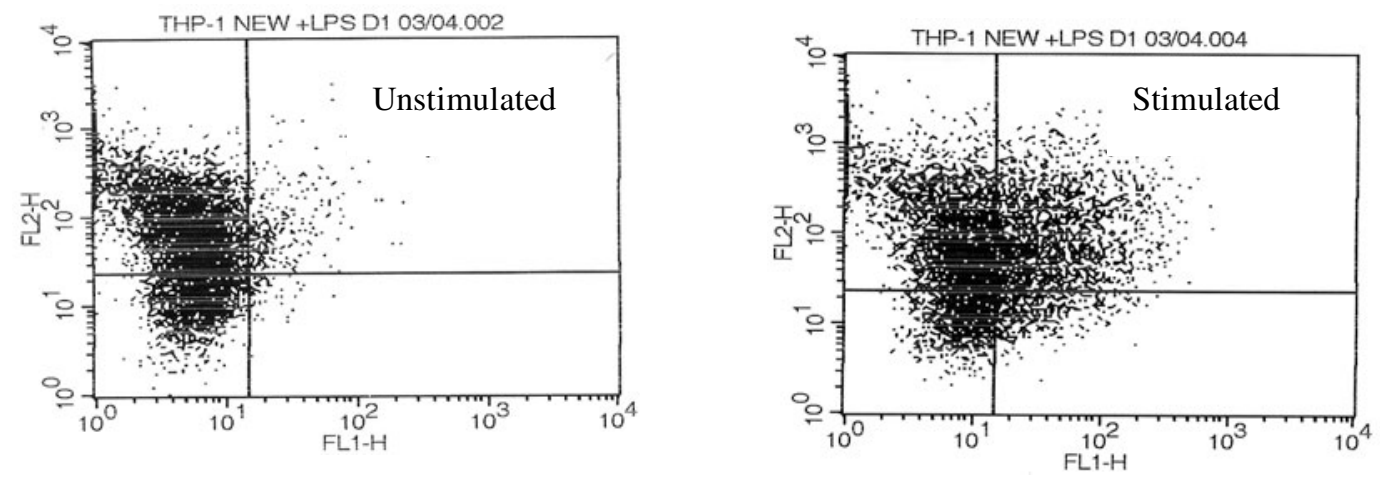

It was found that resting (unstimulated) THP-1 cells did not express CD14, although they expressed variable levels of HLA class II HLA-DR (Figure). The levels of class II showed a marginal increase following stimulation with LPS. CD14 expression was also detected on significant numbers of THP-1 cells following stimulation with LPS (Figure).

1. Hughes DA \& Pinder AC (2000) Am J Clin Nutr. 71, Suppl., 357S-760S. 